High-Performance Computing

\title{
REAL-TIME ROAD SIGNS RECOGNITION USING MOBILE GPU
}

\author{
P.Y. Yakimov \\ Samara National Research University, Samara, Russia
}

\begin{abstract}
This article shows an effective implementation of the algorithm for detection of road signs using video obtained by a camera installed in a vehicle. Road signs detection and recognition are implemented using CUDA and operate in real-time on a mobile GPU. Vehicle speed is used to predict the position of road signs in the adjacent frames in a video sequence. The experimental results confirmed the high efficiency of the developed road signs system.
\end{abstract}

Keywords: road signs recognition, traffic signs recognition, tracking road signs, image processing, mobile computer vision, mobile GPU

Citation: Yakimov PY. Real-Time Road Signs Recognition using Mobile GPU. CEUR Workshop Proceedings, 2016; 1638: 477-483. DOI: 10.18287/1613-0073-2016-1638-477-483

\section{Introduction}

In the past 10 years, the problem of detection of road signs has been solved by many scientists in different countries [1], [2], [3]. The importance of this problem is vital due to road safety, and first studies were started over 30 years ago. However, the power of computer equipment at that time did not allow to carry out frame processing in real time. In addition, digital cameras are not allowed to obtain images of sufficient size.

Recent development of modern mobile processors allowed many car manufacturers to install computer vision systems in automobiles. Among other tasks, such systems also solve the road signs recognition task. However, the main problem of currently provided systems is the low detection accuracy, as well as the inability of some systems to recognize some geographically-specific road signs, for example Russian signs.

This article describes the developed technology to detect and recognize road signs in real-time. The developed system also uses the speed obtained from the vehicle. It makes it possible to predict not only the presence of the object, but also its size and exact location in the adjacent frames. Thus, detection accuracy is improved, while the computational complexity is the same.

To provide a driver with actual information about currently applicable road signs, the system should recognize the localized object. After the localization procedure, a small image of a sign is cut from the original image. The size of this image is scaled to 
$31 \times 31$ pixels, which is enough for recognition. In fact, recognition of objects in such small images does not cause any difficulties, since all possible images of road signs are known in advance. In addition, in the case of successful localization procedures, the exact coordinates of a road sign are known. Thus, the template matching algorithm is used at the classification step. This algorithm shows good results in combination with the localization step.

In order to train and test the developed algorithms, we used the German Traffic Sign Detection Database [4]. Fig. 1 shows several images used to obtain templates for the recognition algorithm and to test the localization method.

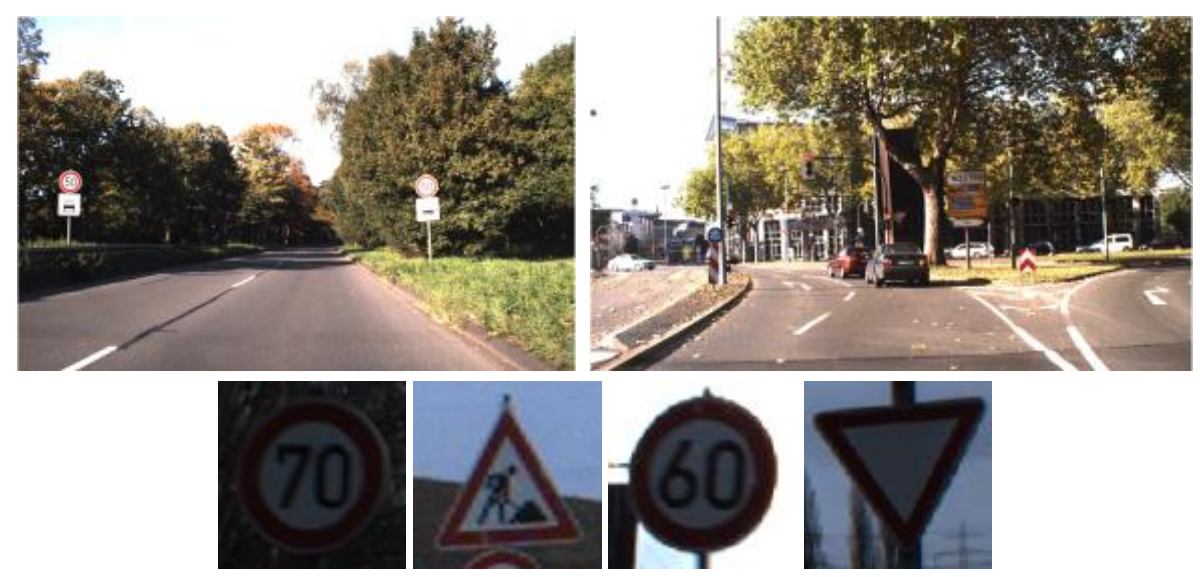

Fig. 1. Images from GTSDB

\section{Road Signs Localization}

Recognition of road signs Is usually implemented in two steps: road sign localization, also known as detection, and its subsequent classification. Most of the detection methods use a single frame from a video sequence to localize the object. This means that they do not use additional information about the sign presence in adjacent frames. Such approaches typically encounter problems with performing in real-time and the detection accuracy. On the other hand, several studies describe tracking algorithms that attempt to predict the location of road signs in the image sequence.

In [5], the authors show that the combination of detection and tracking increases the reliability of the recognition system by reducing the number of false detections. The article [6] shows that tracking helps perform localization faster. However, these algorithms have greater computational complexity and cannot be used in real-time. Paper [7] presents an algorithm for the detection of road signs in video sequences using the current vehicle speed. It allows to predict the exact location of a road sign in the next frame.

The developed technology described in this paper consists of three steps: image preprocessing, the localization of a road sign, and its classification.

To extract red and blue colors from a source image, the HSV color space is used in the preprocessing step. Due to errors of camcorder sensor or small colored objects in 
the images, after applying a threshold filter, some spot noise may occur. To remove this noise, the algorithm described in [8] is applied. Also, paper [8] shows the effective implementation of the noise removal algorithm using massively multi-threaded architecture CUDA. The acceleration using GPUs achieved 60-80 times as compared with conventional implementation on a CPU. The size of a video frame was $1920 \times 1080$ pixels. The CPU-implementation performs in $0.7-1$ second per frame. Using CUDA at NVIDIA GeForce 335M reduced the performing time to 7-10 ms, which satisfies the requirements of video processing in real time.

Paper [9] discusses algorithms for detection and tracking of road signs. The method of localization, which is a modification of the generalized Hough transform, has been developed taking into account the constraints on the executing time of a single video sequence frame processing. The algorithm shows effective results and works well with the preprocessed images. Tracking using the value of the current speed of the car has improved the performance of the system, as the search area in the adjacent frames can be significantly reduced. In addition, the presence of a sign in the sequence of adjacent frames in predicted areas significantly increases the confidence of the detection. Classification of localized road signs will ensure that the whole procedure of recognition of road signs performs successfully.

\section{Road Signs Classification}

To classify the found object, the algorithm uses specially prepared etalon binary images. Fig. 2 shows several such images, which are actually the cut inner area of road signs.

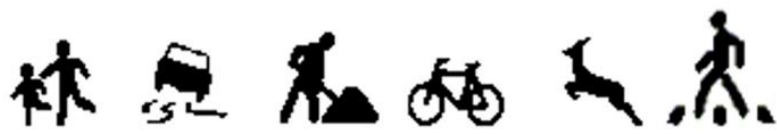

Fig. 2. Etalon images used for classification

For recognition of a sign, a simple subtraction of two images is applied and then the pair with the lowest value is chosen, which will point to the highest proximity to a certain etalon. In the case of large values, the algorithm will give a false detection message, as none of the etalon images suits the localized area. This means that the found the area most likely does not contain a road sign.

The execution time for such classification in average is $1.2 \mathrm{~ms}$ using 32 types of etalon images. Here, each single etalon image type corresponds to a unique image of a road sign. There can be an unlimited number of etalon images of each type in order to consider as many road signs variants as possible. For example, this implementation of the method uses 5 etalon images for every road sign type, for a total of 160 etalon images. 


\section{Experimental results}

To assess the accuracy of the detection algorithm, the German annotated image data base containing images with road signs was used [4]. It contains more than 50000 images of road signs, registered under different conditions. In order to assess the quality of recognition, the overall number of the correctly localized and classified road signs was obtained. When testing the developed algorithms, only 9987 images containing road signs of the required shape and with red outlines were taken into account. The experiments showed $97.3 \%$ correctly localized and classified prohibiting and warning road signs. Table 1 shows the results of accuracy and speed of the algorithms in [4] and the method described in this article.

Table 1. Accuracy and executing time values for road signs recognition algorithms

\begin{tabular}{ccc}
\hline Algorithm & Accuracy & Executing time, FPS \\
\hline $\begin{array}{c}\text { Sliding window + SVM [4] } \\
\text { Modified Generalized Hough } \\
\text { Transform (GHT) } \\
\text { with preprocessing }\end{array}$ & $100 \%$ & 1 \\
$\begin{array}{c}\text { Modified Generalized Hough } \\
\text { Transform (GHT) }\end{array}$ & $89,3 \%$ & \\
without preprocessing & & 25 \\
$\quad$ Viola-Jones [4] & $90,81 \%$ & \\
HOG [4] & $70,33 \%$ & 15 \\
\hline
\end{tabular}

The accuracy of the algorithms listed in the table was obtained using the annotated image database GTSDB [4]. The sliding window algorithm demonstrates the best results in terms of accuracy of road signs recognition $-100 \%$. However, the developed GHT algorithm shows the best executing time. Modification of the generalized Hough transform was also tested in the absence of preprocessing and showed not only lower accuracy, but also almost double the worst executing time.

The modified GHT method was implemented using CUDA technology. Table 2 shows the results of performance improvement. To measure the performance, the mobile processor Nvidia Tegra K1 was used, which contains 192 graphics cores for computation using CUDA technology and 4 CPU cores of ARM architecture.

Table 2. The developed algorithm performance

\begin{tabular}{ccc}
\hline & $\begin{array}{c}\text { Executing time, } \\
\text { ms }\end{array}$ & FPS \\
\hline 1. CPU, 1920x1080 & 441 & 2,27 \\
2. CPU, 1280x720 & 237 & 4,22 \\
3. GPU, 1920x1080 & 33 & 30,07 \\
4. GPU, 1280x720 & 23 & 44,09 \\
\hline
\end{tabular}

The table shows that using CUDA managed to achieve real-time using a mobile GPU. Fig. 3 shows the detection results using the images from GTSDB. Images in Fig. 4 were obtained using a front camera installed into a car. 


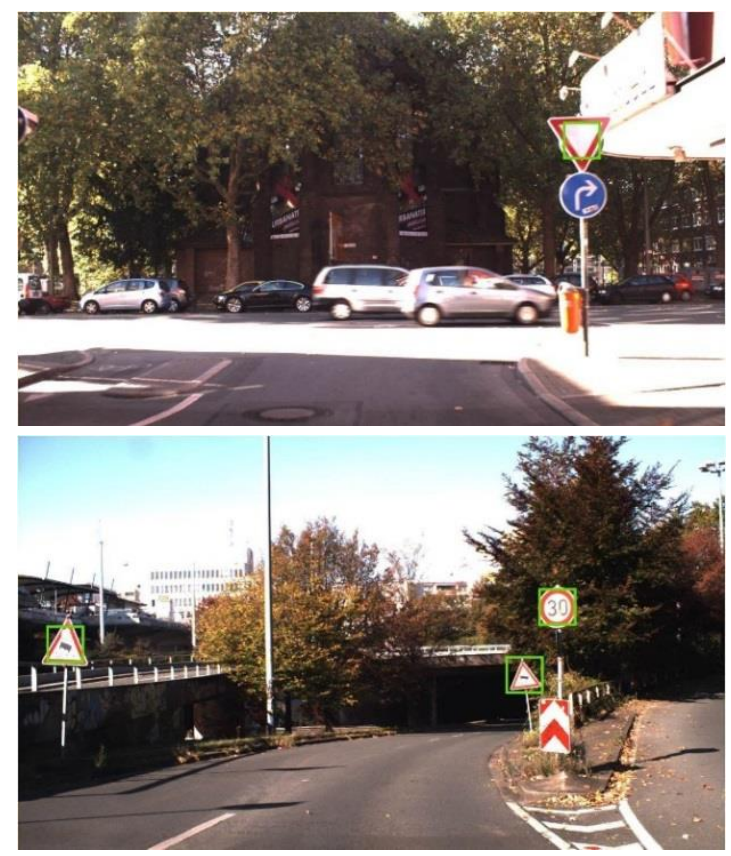

Fig. 3. Localized road signs
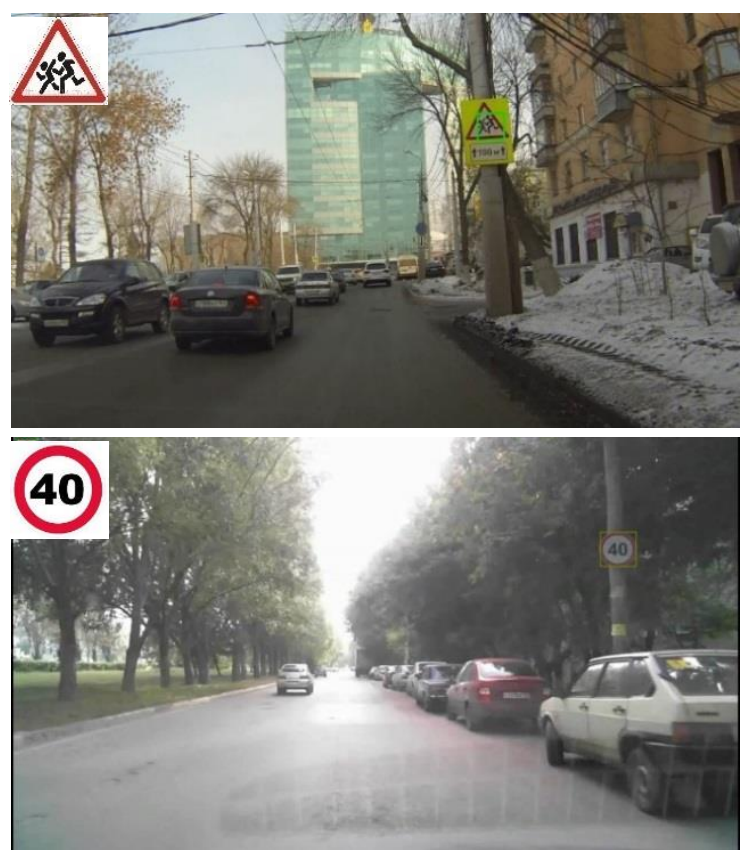

Fig. 4. Localized and recognized road signs 


\section{Conclusion}

The article describes a technology of detection and recognition of road signs. To localize a road sign, a modification of the generalized Hough transform has been developed taking into account the constraints on the executing time of a single frame. The algorithm shows effective results and works well with the preprocessed images. Tracking using the value of the current car velocity has improved the performance of the system, as the search area in the adjacent frames can be significantly reduced. In addition, the presence of the sign in the sequence of adjacent frames in predicted areas significantly increases the confidence of correct detection. Classification of localized road signs ensures that the whole procedure of recognition of road signs is successful. Testing the described algorithms using video sequences has proved the ability to process high-resolution video stream in real-time. The detection range of road signs when using a FullHD camera is up to $50 \mathrm{~m}$.

Massively multithreaded programming technology CUDA was used to speed up the implementation of the developed algorithms. When using a mobile GPU Nvidia Tegra $\mathrm{K} 1$, the acceleration reaches 15 times.

\section{Acknowledgements}

This work was supported by Project \#RFMEFI57514X0083 by the Ministry of Education and Science of the Russian Federation.

\section{References}

1. Shneier M. Road sign detection and recognition. Proc. IEEE Computer Society Int. Conf. on Computer Vision and Pattern Recognition, 2005: 215-222.

2. Ruta A, Porikli F, Li Y, Watanabe S, Kage H, Sumi K. A New Approach for In-Vehicle Camera Traffic Sign Detection and Recognition. IAPR Conference on Machine Vision Applications (MVA), Session 15: Machine Vision for Transportation, 2005.

3. Belaroussi R, Foucher P, Tarel JP, Soheilian B, Charbonnier P, Paparoditis N. Road Sign Detection in Images. 20th International Conference on Pattern Recognition (ICPR), 2010: 484-488.

4. Houben S, Stallkamp J, Salmen J, Schlipsing M, Igel C . Detection of Traffic Signs in Real-World Images: The German Traffic Sign Detection Benchmark. International Joint Conference on Neural Networks, 2013.

5. Lafuente-Arroyo S, Lafuente-Arroyo S, Maldonado-Bascon S, Gil-Jimenez P, GomezMoreno H, Lopez-Ferreras F. Road sign tracking with a predictive filter solution. IEEE 32nd Annual Conference on Industrial Electronics, 2006: P. 3314-3319.

6. Lopez L, Fuentes O. Color-based road sign detection and tracking. Image Analysis and Recognition. Lecture Notes in Computer Science, Springer, 2007: 1138-1147.

7. Yakimov PY. Tracking traffic signs in video sequences based on a vehicle velocity. Computer Optics, 2015; 39(5): 795-800. DOI: 10.18287/0134-2452-2015-39-5-795-800.

8. Yakimov PY. Preprocessing of digital images in systems of location and recognition of road signs. Computer Optics, 2013; 37(3): 401-405. 
9. Yakimov PY. Localization of objects contours with different scales in images using Hough Transform. Computer Optics, 2013; 37(4): 496-502.

10. Mathias M, Timofte R, Benenson R, Gool LV. Traffic sign recognition - how far are we from the solution? Proceedings of IEEE International Joint Conference on Neural Networks, 2013: 1-8. 